\title{
Introduction aux lois de comportement des sables très peu denses
}

\author{
Introduction to very loose sand behaviour laws
}

J. BIAREZ, F. ZIANI

Ecole Centrale de Paris*

Rev. Franç. Géotech. n 54, pp. 65-73 (janvier 1991)

\section{Résumé}

Après avoir montré que le comportement des sables très peu denses est analogue à celui des argiles normalement consolidées et cherché à définir une contrainte de consolidation fictive pour les sables sans écrasement de particules, on a montré qu'en première approximation, on pourrait formuler des lois de comportement pour les sables analogues à celles issues des travaux de ROSCOE pour les argiles.

\footnotetext{
Abstract

After having showed that the behaviour of very loose sand is analogous to that of the normally consolidated clay and having tried to define a fictive consolidation stress for sand without particle crushing, we have shown in a first step that we could formulate a behaviour law for sands similar to those issued from ROSCOE's works for clays.
} 


\section{NOTATIONS}

$\sigma_{1}^{\prime}, \sigma_{2}^{\prime}, \sigma_{3}^{\prime}$; contraintes principales ;

$\mathrm{q}=\sigma_{1}^{\prime}-\sigma_{3}^{\prime}:$ déviateur $;$

$\mathrm{p}=\frac{\sigma_{1+}^{\prime} \sigma_{2+}^{\prime} \sigma_{3}^{\prime}}{3}:$ contrainte moyenne ;

$\epsilon_{1}$ : déformation axiale ;

$e:$ indice des vides:

$\gamma_{\mathrm{d}}$ : poids volumique $\mathrm{sec}$ :

$e_{\max }:$ maximum ;

$e_{\min }:$ minimum ;

$\sigma_{\text {iijic }}$ : tenseur de contrainte de consolidation fictif ;

P'ic : pression fictive de consolidation.

\section{INTRODUCTION}

Cette étude fait suite à celle présentée au colloque NSF-CNRS de Cleveland [3] indiquant les hypothèses retenues pour une première approche commune aux sables et aux argiles remaniées.

Le but de cet article est de présenter des résultats expérimentaux montrant que les sables extrêmement peu denses ont un comportement du même type que celui des argiles normalement consolidées et que les sables usuels plus denses ont une analogie avec les argiles surconsolidées.

Ainsi on est amené à définir une contrainte de consolidation dite fictive pour les sables usuels sans écrasement de particules, une élasticité non linéaire pour les petites déformations $\left(\epsilon_{1}<10^{-5}\right)$ et une limite pseudo-élastique volumétrique comme pour les argiles.

Pour atteindre les très faibles densités, nous avons utilisé un sable légèrement humide dont les caractéristiques sont :

$$
\begin{aligned}
& \begin{array}{l}
\mathrm{d} 60=0,3 \mathrm{~mm} ; \\
\mathrm{d} 10=0,6 \mathrm{~mm}:
\end{array} \\
& \gamma \mathrm{d} \min =14,5 \mathrm{kN} / \mathrm{m}^{3} ; \text { e } \max =0,84 \\
& \gamma \mathrm{d} \max =16,5 \mathrm{kN} / \mathrm{m}^{3} ; \text { e } \min =0,62
\end{aligned}
$$

Les manipulations sur un sable très peu dense étant difficiles, il ne faut en retenir que les aspects qualitafifs.

Par ailleurs, des essais de force portante de fondation ont été réalisés sur ces sables très peu denses pour obtenir une logique générale pour ce sujet quelque soit la densité. Cette étude entre dans nos recherches sur la locomotion tous terrains en particulier le compactage provoqué par les engins agricoles et les résultats ont été publiés dans la revue Journal of Terramechanics [7].

\section{PLASTICITÉ PARFAITE ET INDICE DES VIDES "CRITIQUE *}

Les lois de comportement ne peuvent être facilement obtenues que dans des essais où la déformation est homogène. Malheureusement, dans les essais triaxiaux, il se crée souvent des localisations de déformation qui conduisent à des discontinuités cinématiques. Celles-ci produisent l'arrêt de la dilatance dans les plans $\left(e-\epsilon_{1}\right)$ et $\left(e-p^{\prime}\right)$. La figure 1 rappelle que l'on peut limiter le rôle de ces localisations en utilisant des échantillons de petite hauteur relative $\mathrm{H} / 2 \mathrm{R}$ $=0,5$ avec antifrettage [8]. On obtient ainsi des relations différentes dans le plan $\left(e-p^{\prime}\right)$ suivant que l'échantillon est de grande hauteur relative $\mathrm{H} / 2 \mathrm{R}=$ 2,2 (fig. 2), ou de petite hauteur relative $\mathrm{H} / 2 \mathrm{R}=$ 0,5 (fig. 3) mais il est difficile d'être certain d'atteindre la plasticité parfaite.

La solution consiste à rechercher une borne supérieure des valeurs $\left(e-p^{\prime}\right)$ avec des essais normalement consolidés. La représentation sous forme de cinq figures associées facilite l'analyse de la cohérence des résultats et permet de contrôler sur la cinquième dans le plan $\left(e-\log p^{\prime}\right)$ que la plasticité parfaite est bien parallèle aux compressibilités isotrope et œdométrique dans le domaine de contrainte courant.

Nos expériences montrent que les sables peu denses présentent bien des résultats analogues aux argiles normalement consolidées (fig. 4).

La figure 5 indique que l'on peut atteindre la plasticité parfaite par des essais dilatants habituels mais aussi par des essais contractants ce qui permet d'obtenir une borne inférieure et une borne supérieure pour la relation fondamentale entre l'indice des vides et la contrainte moyenne en plasticité parfaite. Ces bornes sont voisines des résultats obtenus sur un sable de densité intermédiaire.

Les sables très peu denses donnent probablement des indices des vides trop forts étant donné certaines difficultés expérimentales pour mesurer l'indice des vides initial dans l'essai triaxial.

Pour les sols remaniés, on constate en général que la droite de pente $\mathrm{M}$ du palier sépare les domaines contractant et dilatant comme l'ont indiqué ROSCOE pour les argiles [5] et LUONG pour les sables [4].

\section{CHEMIN OEDOMÉTRIQUE}

La compression des sables extrêmement peu denses se traduit par une déformation plastique représentée par une droite, dans le plan (e-log p') (fig. 6), parallèle aux chemins isotropes et de plasticité parfaite précédents. Si on appelait $\mathrm{Cc}$ la pente de cette droite, on constaterait qu'elle est du même ordre de grandeur que celle des argiles pour les faibles limites d'Atteberg.

Pour les fortes contraintes (10 MPa), la courbe descend sous cette droite en raison de l'écrasement des particules. Par ailleurs, on a montré que l'indice des vides « critique " dépendait de $\frac{\mathrm{d} 60}{\mathrm{~d} 10}$ et qu'il était voisin de $e_{\max }$ pour une pression moyenne de l'ordre de $0,1 \mathrm{MPa}[6]$.

Le déchargement œedométrique suit un chemin beaucoup plus réversible que le chargement. On pourrait comparer la pente au $\mathrm{Cs}$ de l'argile mais il est plus 

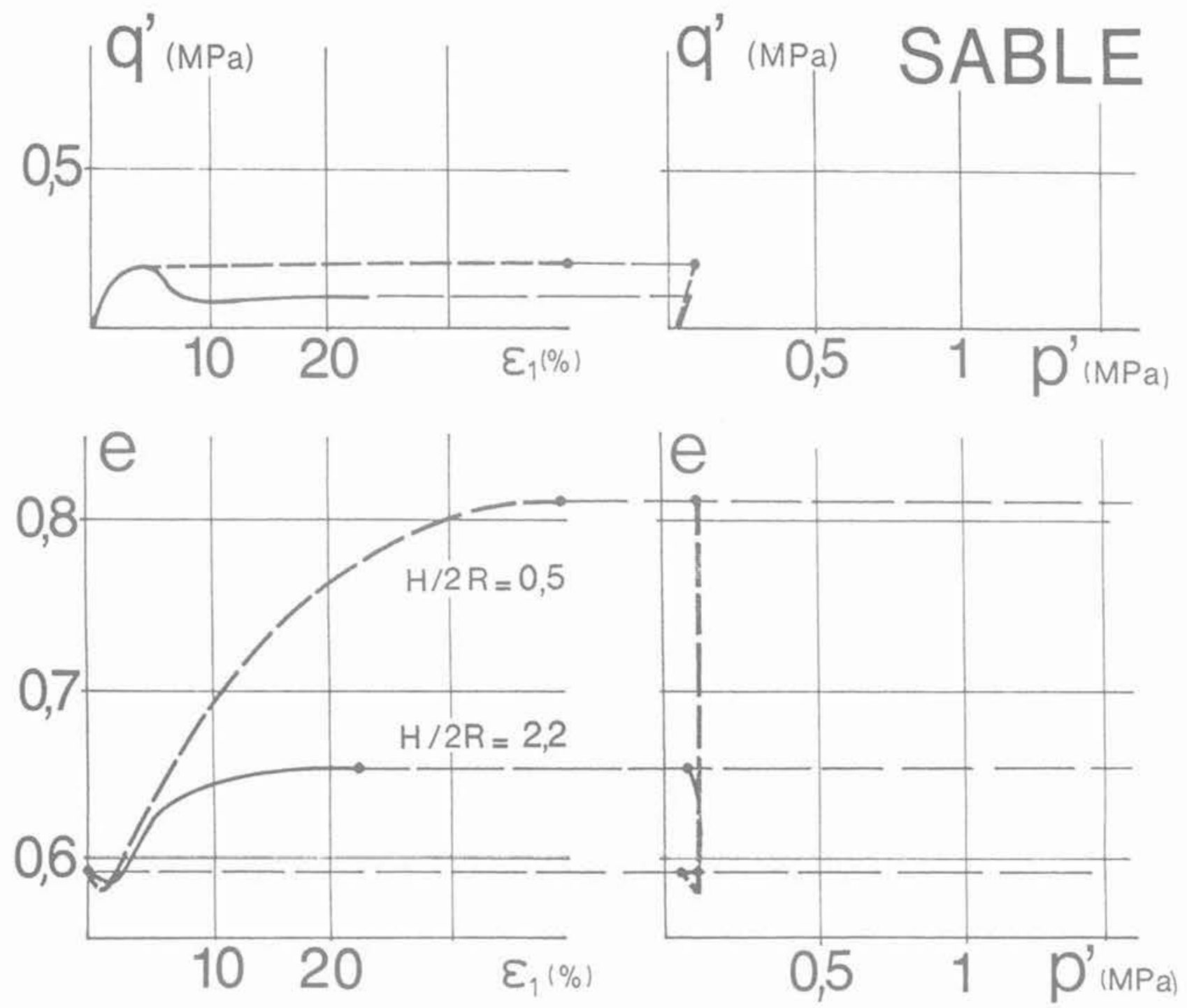

Fig. 1. - Essais triaxiaux sur sable saturé de différentes hauteurs.

Fig. 1. - Triaxial tests on saturated sand of different heights.

logique de retenir la loi élastique non linéaire de Hertz:

$$
E i=\frac{3}{2}\left[\frac{4}{3 K(e)} \cdot \frac{E g}{1-\nu^{2} g}\right]^{2 / 3} \cdot p^{1 / 3}
$$

où :

- Eg et $\nu_{\mathrm{g}}$ représentent respectivement le module de Young du matériau et le coefficient de Poisson ; - K(e) est une fonction qui dépend de l'indice des vides ;

- P est la pression moyenne:

- Ei est le module d'élasticité isotrope.

L'exposant $1 / 3$ est à remplacer par $1 / 2$ si l'on remplace les sphères régulièrement arrangées par du sable courant. Ces valeurs se retrouvent dans des essais triaxiaux à faible déformation $\left(\epsilon_{1}<10^{-5}\right)$ (fig. 7) ou dans des essais de résonance. Il en est de même pour les argiles [3].
Par analogie avec les essais cedométriques sur l'argile. on est tenté de parler d'une contrainte limite élastique ou plus généralement d'un tenseur de contrainte de consolidation $\sigma$ 'ijic pour tenir compte de l'anisotropie.

Pour les essais sur sables de densité élevée sans rupture de particules, on est amené à définir une contrainte fictive de consolidation P'ic sur le prolongement de la droite de pente $\mathrm{Cc}$ précédente.

Cette valeur est dite fictive pour les sables courants car elle conduirait à l'écrasement des grains si l'on suivait le chemin œdométrique pour atteindre les densités usuelles in situ. Mais elle est indispensable pour la formulation de la loi de comportement commune aux sables et aux argiles pour les chemins à faible contrainte sans écrasement de particules. 

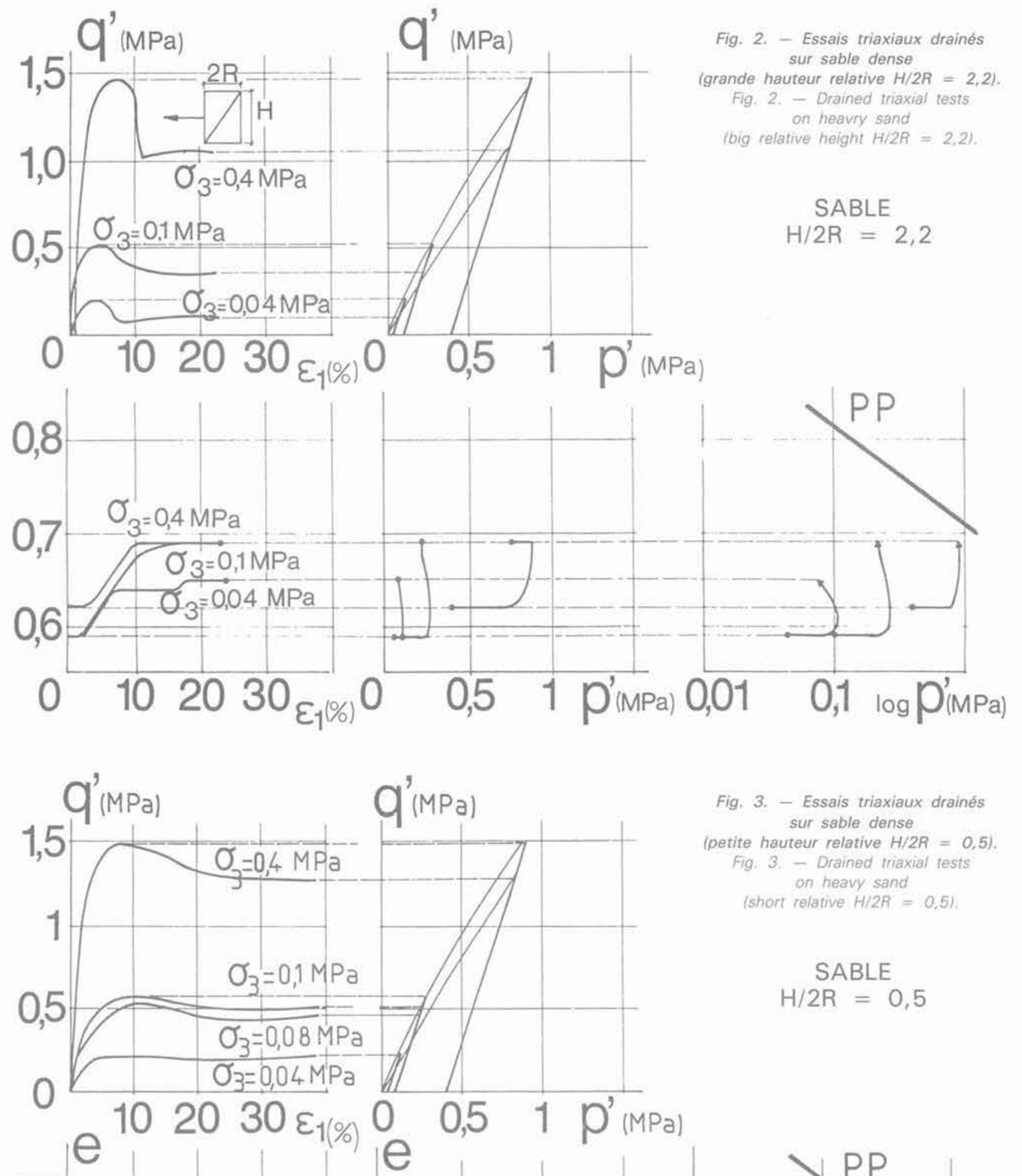

Fig. 3. - Essais triaxiaux drainés sur sable dense (petite hauteur relative $H / 2 R=0,5$ )

Fig. 3. - Drained triaxial tests on heavy sand

(short relative $H / 2 R=0,5$ )

SABLE

$\mathrm{H} / 2 \mathrm{R}=0,5$


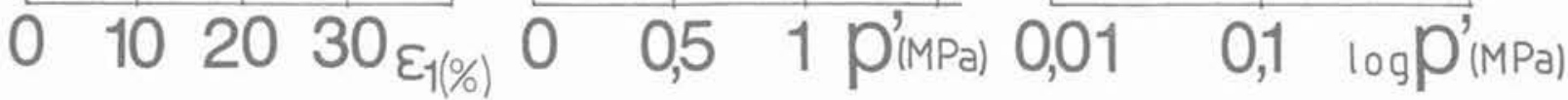



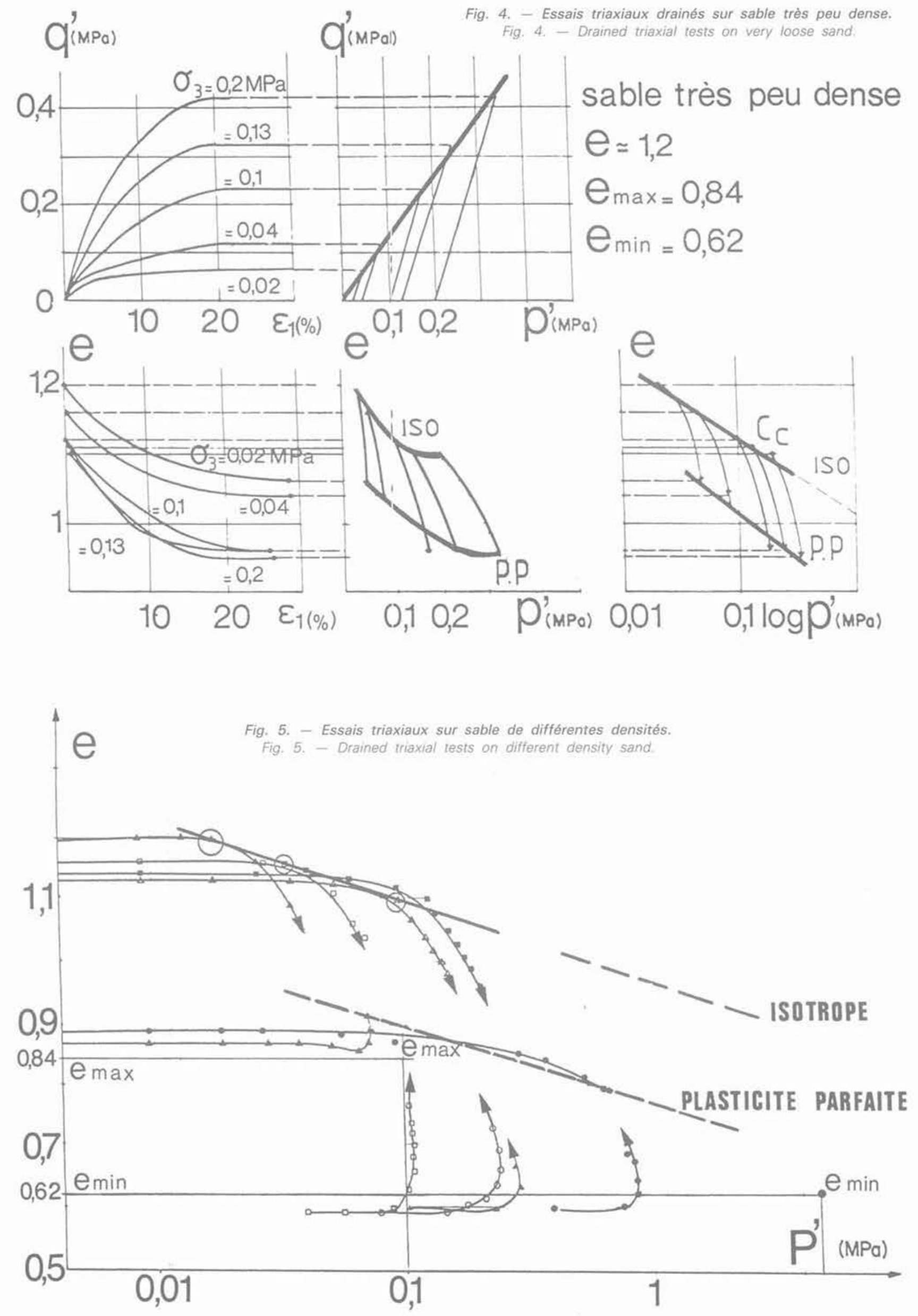


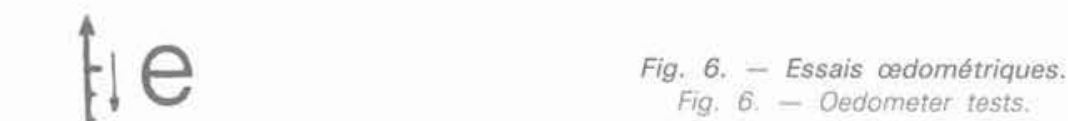

Fiq. 6. - Oedometer tests.
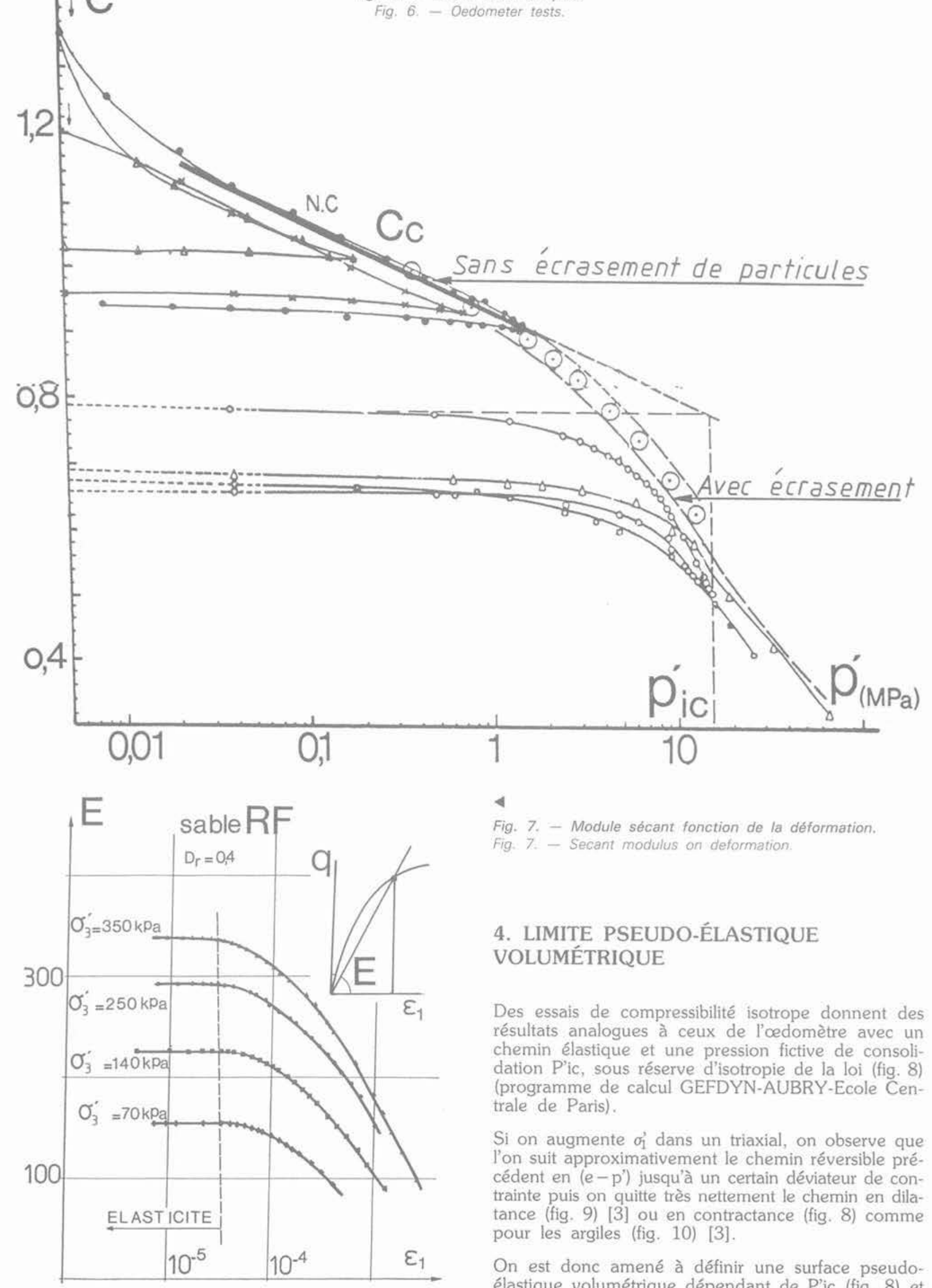

Fig. 7. - Module sécant fonction de la déformation.

Fig. 7. - Secant modulus on deformation.

\section{LIMITE PSEUDO-ÉLASTIQUE VOLUMÉTRIQUE}

Des essais de compressibilité isotrope donnent des résultats analogues à ceux de l'œedomètre avec un chemin élastique et une pression fictive de consolidation P'ic, sous réserve d'isotropie de la loi (fig. 8) (programme de calcul GEFDYN-AUBRY-Ecole Centrale de Paris).

Si on augmente $\sigma_{1}$ dans un triaxial, on observe que l'on suit approximativement le chemin réversible précédent en ( $e-p$ ') jusqu'à un certain déviateur de contrainte puis on quitte très nettement le chemin en dilatance (fig. 9) [3] ou en contractance (fig. 8) comme pour les argiles (fig. 10) [3].

On est donc amené à définir une surface pseudoélastique volumétrique dépendant de P'ic (fig. 8) et 


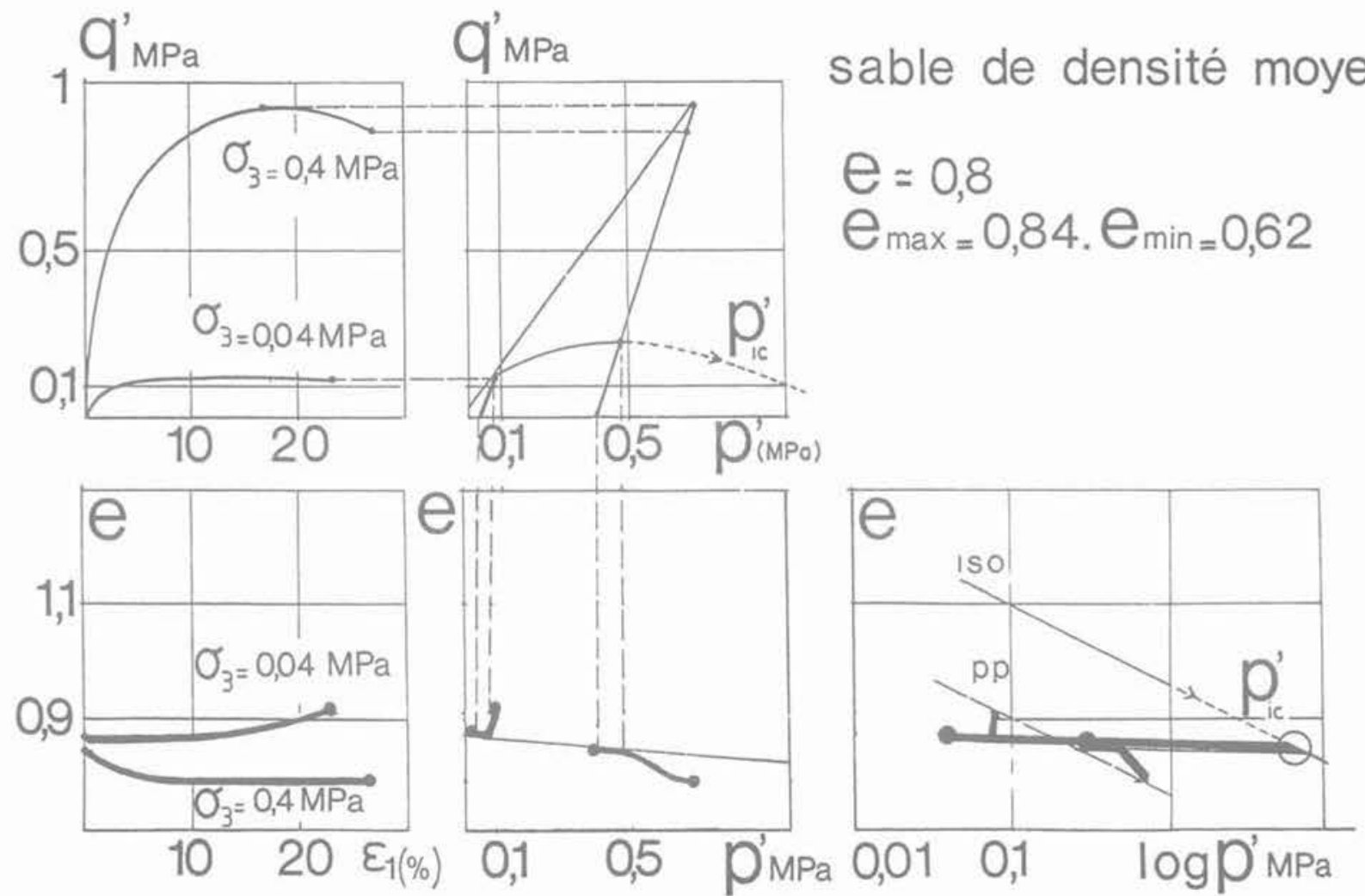

Fig. 8. - Essais triaxiaux drainés sur sable de densité moyenne

Fig. 8. - Drained triaxial test on average density sand.

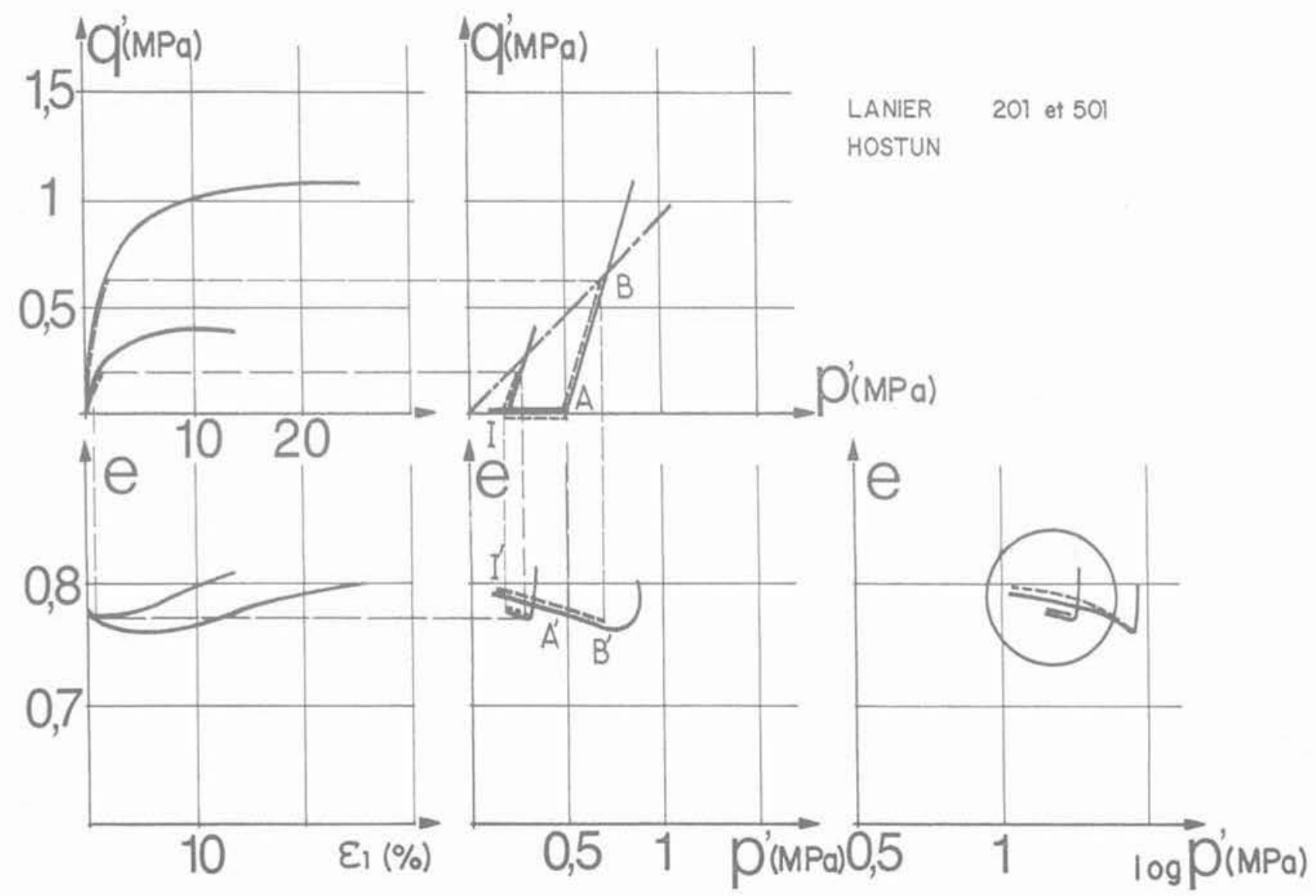

Fig. 9. - Essais triaxiaux drainés sur sable d'Hostun.

Fig. 9. - Drained triaxial tests on Hostun's sand. 


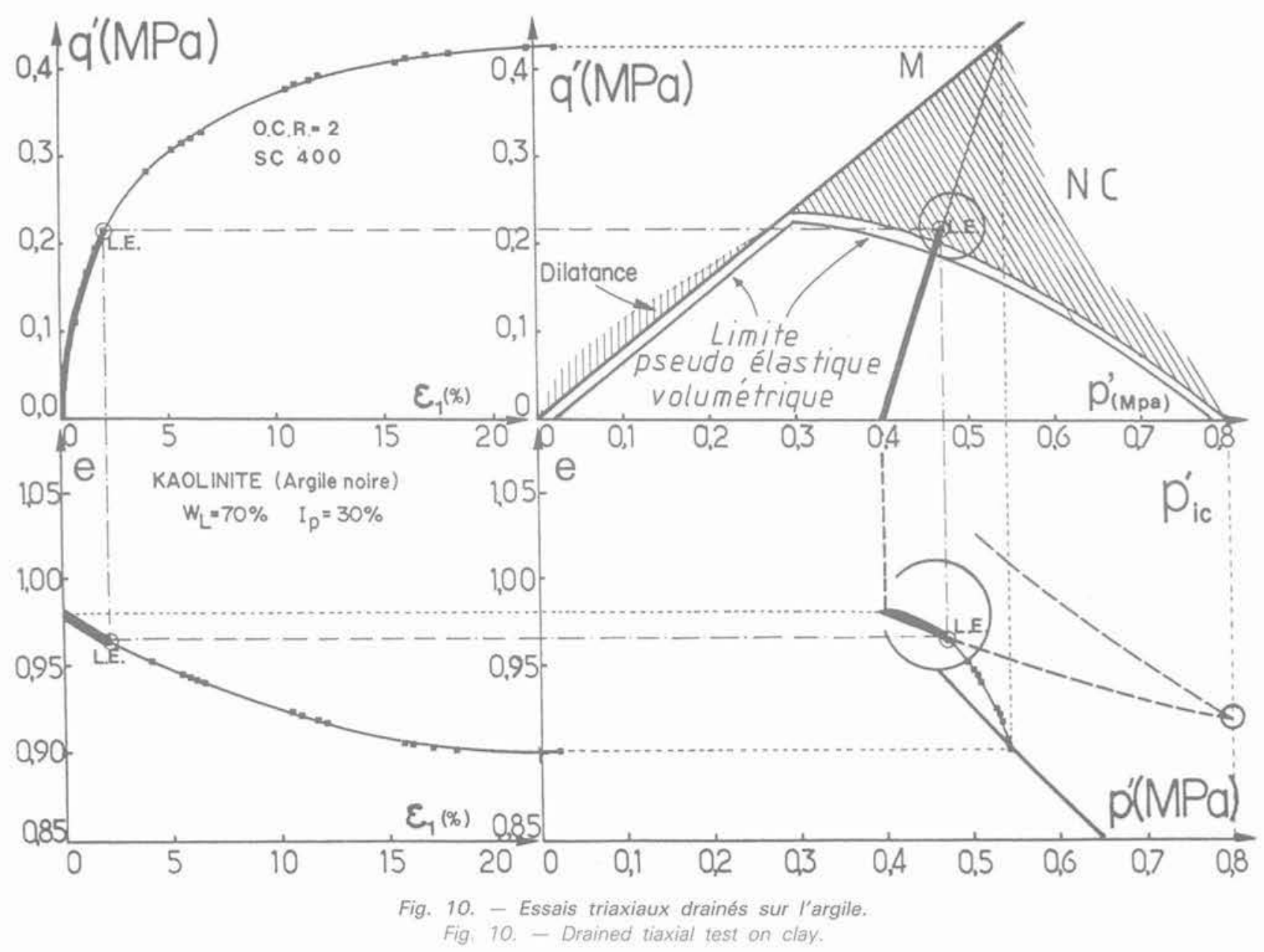

plus généralement d'un tenseur de contrainte de surconsolidation $\sigma$ 'jic pour le cas usuel d'anisotropie.

En pratique, cette anisotropie plus une légère cimentation entre les grains, courante dans les sols non remaniés, conduit à une résistance maximale des sols surconsolidés voisine de cette surface limite élastique volumétrique.

Pour les calculs pratiques de remblais sur les sols normalement consolidés, on sort rapidement de cette surface ; on peut donc négliger les aspects non linéaires et non réversibles intérieurs à cette surface.

\section{CONCLUSION}

Les essais triaxiaux effectués sur les sables extrêmement peu denses mettent en évidence une densification analogue à celle des argiles normalement consolidées. Les valeurs finales sont approximativement alignées sur une droite dans l'espace $(e-\log p$ ') voisine de celle des sols surconsolidés.

En essayant de définir un tenseur fictif de consolidation dont dépend une surface pseudo-élastique volu- métrique, il serait possible de faire les mêmes hypothèses simplificatrices et donc de formuler les mêmes lois de comportement pour les argiles et les sables.

Evidemment pour les argiles, il faut faire intervenir les dérivées par rapport au temps des tenseurs contrainte et déformation pour expliquer les phénomènes liés à la « consolidation secondaire ...

\section{BIBLIOGRAPHIE}

[1] J. BIAREZ et al. (1969), Propriétés mécaniques des sols sous diverses sollicitations. Proc. 7th Int. Soil Mech. Montréal Vol. 1, pp. 165-169.

[2] J. BIAREZ, B. CAMBOU (1971), Compressibilité des milieux pulvérulents sur chemin isotrope ou cedométrique et rupture des particules. Bulletin spécial des laboratoires des Ponts et Chaussées, 17-19 mai 1971.

[3] J. BIAREZ, P.Y. HICHER (1987). Simplified hypotheses on mechanical properties equally applicable to sands and clays. Conference introductive à International Workshop in Constitutive Equations for granular non cohesive soils. July, 1987, Cleveland USA. 
[4] M.P. LUONG (1980), Phénomènes cycliques dans les sols pulvérulents. Revue Française de Géotechnique $\mathrm{n}^{\circ} 10$, pp. 39-53.

[5] ROSCOE K.H. (1958), Shofield A.N. and C.P. Wroth. On the yielding of soils. Géotechnique n०8, pp. 22-53.

[6] ZERVOYANIS (1982), Etude synthétique des propriétés mécaniques des argiles saturées et des sables sur chemin cedométrique et triaxial de révolution. Thèse de Docteur-Ingénieur. Ecole Centrale de Paris.

[7] F. ZIANI, J. BIAREZ (1990), Pressure-sinkage relationship for tyres on very loose sand.Journal of terramechanics vol. $27, \mathrm{n}^{\circ} 3$.

[8] F. ZIANI (1987), Contribution à l'étude du com. pactage des sols : cas particulier du comportement des sables très peu denses. Thèse de Doctorat. Faculté des Sciences de Gembloux. 